LOGARITHMIC GRAPHS DISTORT JUDGMENT

Versions: this preprint was submitted for peer review on 5/4/2020 and version 1 was published online on 5/19/2020. The preprint was subsequently accepted for publication at Behavioral Science \& Policy, and version 2 was published online on 10/6/2020.

\title{
Logarithmic Axis Graphs Distort Lay Judgment
}




\section{Summary}

COVID-19 data is often presented using graphs with either a linear or logarithmic scale. Given the importance of this information, understanding how choice of scale changes interpretations is critical. To test this, we presented laypeople with the same data plotted using differing scales. We found that graphs with a logarithmic, as opposed to linear, scale resulted in laypeople making less accurate predictions of growth, viewing COVID-19 as less dangerous, and expressing both less support for policy interventions and less intention to take personal actions to combat COVID-19. Education reduces, but does not eliminate these effects. These results suggest that public communications should use logarithmic graphs only when necessary, and such graphs should be presented alongside education and linear graphs of the same data whenever possible. 
LOGARITHMIC GRAPHS DISTORT JUDGMENT

The current global COVID-19 epidemic has resulted in citizens informing themselves through print and social media. Journalists and epidemiologists need to decide how to present their data in a way that facilitates understanding by the lay audience. Even though the objective reality of the data may be identical, different presentations of the data could seriously influence interpretations (see figure 1). Being aware of how different presentation choices influence interpretation is critical because lay people incorrectly interpreting data may affect their beliefs about how necessary it is to take precautions to protect themselves and others ${ }^{1}$.

Confirmed cases (linear scale)

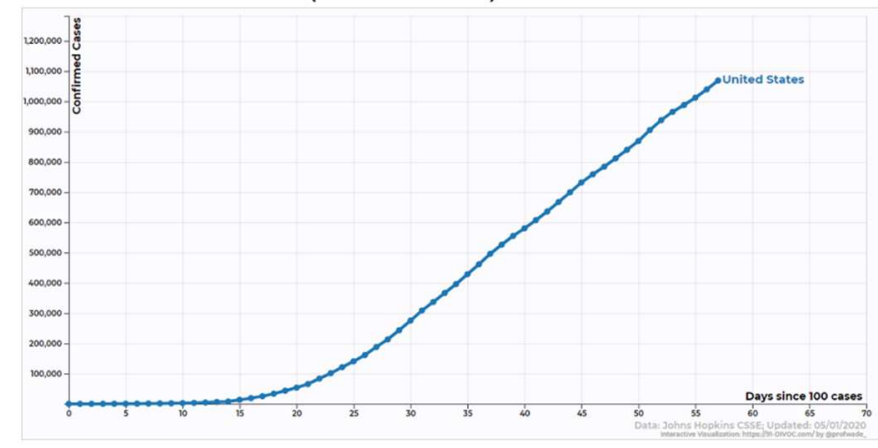

Confirmed cases (logarithmic scale)

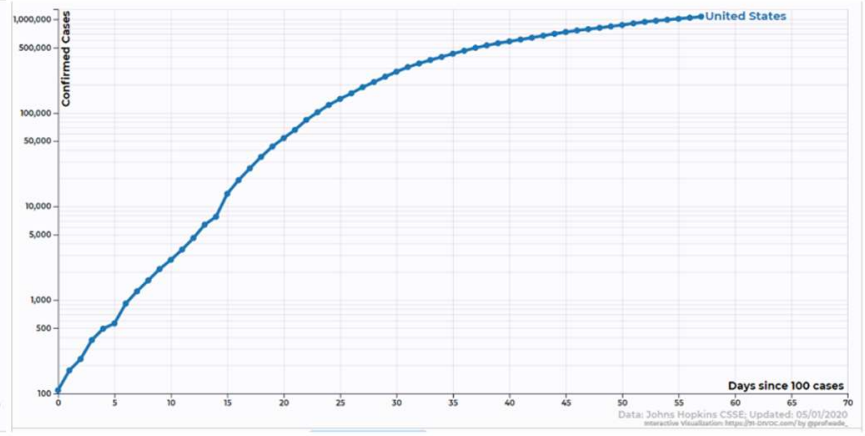

Figure 1. These graphs show the number of confirmed Coronavirus cases in the US over time with either a linear (left) or logarithmic (right) scale y axis as represented by 91-divoc.com, and clearly demonstrates the difference in subjective appearance between the two scales (data presented as of 05/01/2020). Similar graphs were used in Study 1a. This data visualization website has been widely shared virally online, and has been used in briefings by the Governors of Kentucky ${ }^{i}$ and Washington ${ }^{i i}$. Titles have been added above plots for clarity.

When presenting data on disease growth, scientists generally use either a logarithmic or a linear y-axis. The logarithmic y-axis takes the logarithm of values, which has the effect of making each interval on the graph correspond to an order of magnitude increase in values. Logarithmic axes are useful because these graphs make it easier to compare exponential growth-rates and see how they change over time.

\footnotetext{
${ }^{\mathrm{i}} \mathrm{https}: / / \mathrm{www} \cdot$ youtube.com/watch?v=gSzUuOTzGE8\&feature=youtu.be $\& \mathrm{t}=1595$

ii https://twitter.com/GovInslee/status/1243237794895413249
} 
LOGARITHMIC GRAPHS DISTORT JUDGMENT

They are also helpful when comparing countries with cases that are an order of magnitude different from one another - for example, on a linear graph if one country has 10,000 cases country and a second has only 100 cases, the second country would take up so little of the graph that it would be unreadable. In contrast, linear graphs more directly present the actual case count and avoid the danger of interpretations being distorted by misinterpreted axes. Thus, which y-axis is appropriate depends on which information a graph aims to convey.

Evidence suggests that outlets use both types of graphs. We reviewed graphs in three major newspapers ${ }^{\mathrm{iii}}$ and found that while linear scales were most common in articles, every publication also used logarithmic scales . Notably, the Financial Times' presented its primary COVID-19 tracker in a logarithmic format. Additionally, laypeople increasingly access scientific communications directly through social media and preprint services ${ }^{2 \mathrm{iv}}$, where logarithmic graphs are omnipresent. Here, we investigate lay audience's interpretations of these graphs.

While using either a linear or a logarithmic y-axis does not affect the underlying data, it is likely that individuals are affected by data's presentation. First, individuals are notoriously bad at numerical thinking. Numerical thinking is often measured using an objective numeracy scale and between 16-20\% of participants fail to answer the simplest questions on the scale correctly ${ }^{3}$. Because logarithmic scales require relearning the meaning of different graphical patterns based on a new, mathematically transformed scale, individuals may not be able to appropriately extrapolate from them. This is especially likely with the exponential growth often found in disease data ${ }^{4,5}$. Even if individuals are able to correctly interpret logarithmic scales, they may not be motivated to do so and instead prefer to avoid numeric computations, a tendency encapsulated by the subjective numeracy scale ${ }^{6,7,8}$. Rather than relying on active computation, individuals often use intuitive, system 1-like judgments that are more likely to be biased ${ }^{9}$. As a consequence, their judgments are influenced by logically irrelevant design choices ${ }^{10}$. For example,

\footnotetext{
iii We reviewed the Financial Times, New York Times, and Wall Street Journal. Full details on this search can be found in the Appendix.

iv E.g. medical preprint site medRXiv saw its visitors increase 100-fold since December
} 
LOGARITHMIC GRAPHS DISTORT JUDGMENT

identical data in the same type of graph can yield different inferences of growth-rates if the aspect-ratio of the graph is changed to make the graph's slope appear more or less steep ${ }^{11,12}$, and other research has found that even many experts can misunderstand graphs with nonstandard design choices ${ }^{13}$. All of this suggests that consumers of COVID-19-related data may tend to underestimate the severity of the pandemic when that data is presented using a logarithmic scale.

In four studies we investigated the accuracy of predictions based on each type of graph, perceptions of threat, and attitudes toward government and individual action. Please refer to the Appendix for additional details on these studies' methods, results, and supplementary analyses, as well as details on three additional studies. Based on reviewer suggestions, in addition to the Frequentist analysis reported in the main paper we carried out non-preregistered Bayesian analyses which replicate our primary findings, and can be found in the Appendix. Full materials, preregistrations, and data can be found at https://osf.io/zqut5/?view_only=3aa66d592dd2495ca508b4fa8729381a.

In short, we find that participants are reliably less accurate in their predictions of growth and believe COVID-19 is less dangerous when data is presented using a logarithmic rather than a linear scale (figure 2). Additionally, this has downstream consequences on attitudes towards protective behavior by the government and the self.

\section{S1: Predictions}

In Study 1, we tested whether graphs using either logarithmic or linear scales affected the accuracy of individual's predictions of future growth. In Study 1A participants used graphs of real countries' total Coronavirus cases to predict actual case counts in the future ${ }^{v}$. Since it was possible that a country changed the criteria for counting their number of cases during this study, we also ran Study 1B, a

\footnotetext{
${ }^{v}$ Actual case counts were derived from the COVID-19 data repository maintained by the Center for Systems Science and Engineering at John Hopkins University: https://github.com/CSSEGISandData/COVID-19
} 
LOGARITHMIC GRAPHS DISTORT JUDGMENT

conceptual replication in which we presented participants with hypothetical growth data and compared their predictions to the number of cases we would expect based on these hypothetical growth curves.

Participants. 266 Mechanical Turk (MTurk) workers $\left(\mathrm{M}_{\mathrm{age}}=38\right.$ years, $40 \%$ female) completed Study 1A. 403 MTurk workers $\left(\mathrm{M}_{\mathrm{age}}=36.7\right.$ years, 37\% female $)$ completed Study 1B.

Procedure. Both studies involved two conditions; one in which the graphs were presented with a logarithmic scale, and one in which linear scales were used (see figure 1). For each country (four in Study $1 \mathrm{~A}$ and one in 1B) participants predicted the number of cases in one, three, five, and ten days from the present. We also collected judgments of the growth and danger of the hypothetical disease from participants - these results are shown in figure 5, and the measures are discussed in more detail in Studies 2 and 3.

Results. Overall, the mean absolute error in participant's predictions was higher in the logarithmic axis condition than the linear axis condition (see figure 3 ). We found the same effect for the more controlled hypothetical study. 

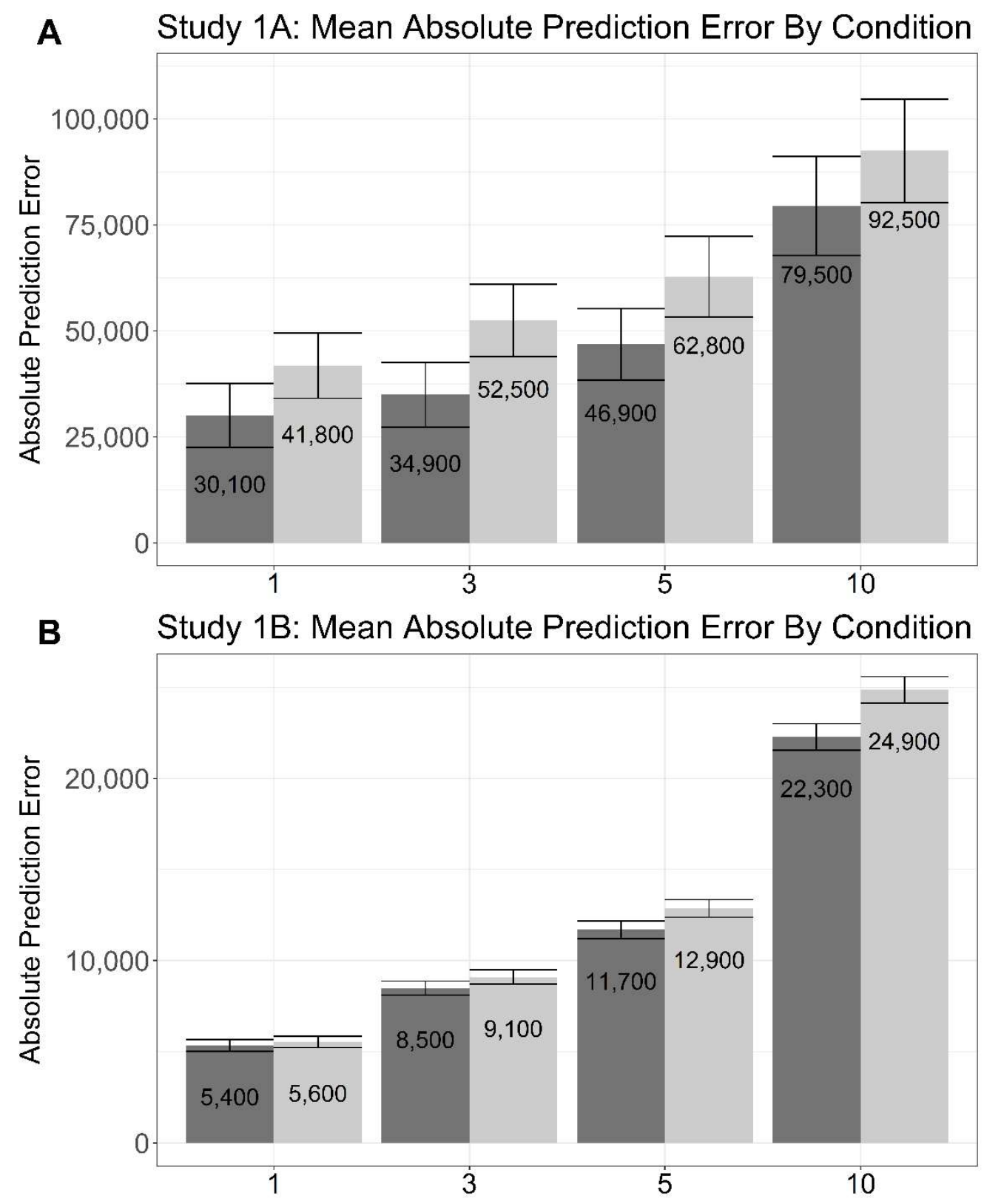

Scale Condition

Linear Scale Logarithmic Scale

Figure 2. The mean absolute prediction error by condition for Study $1 A(A$, left) and Study $1 B$ (B, right).

For each prediction the error is greater in the logarithmic condition across both studies. Error bars on the figure are calculated as the population-level standard error for the measure, here and in all following figures. 
LOGARITHMIC GRAPHS DISTORT JUDGMENT

Taken together, these results suggest that logarithmic scales tend to make laypeople's judgments about growth less accurate by increasing the variance in individual's predictions. It is possible that this difference is due to the lower granularity in the logarithmic scale. At the values above 10 where predictions are being made the same physical distance corresponds to a greater absolute change in cases on a logarithmic scale axis than a linear one, which may drive this greater variance in participant's results. However, this isn't the only way to measure accuracy. One could also ask whether presenting the data in a log or linear format creates systematic over- or under-estimation among the population - not only increasing the inconsistency in peoples judgments, but actually biasing most people in one direction or the other. To test this, we conducted alternative analyses looking at biases at the group level (average over- and underestimation). These results are inconsistent across studies, likely due differences between graphs used and the higher variability of this measure. In general, within these studies we don't find a link between accuracy of participant's case count predictions and their subjective judgments or behavioral responses, although it is possible that such a connection may exist but we do not have the statistical power to detect it. Additional details on these accuracy analyses for these and following studies can be found in the appendix. Note that looking at accuracy of population level predictions is risky - for example, if some people overestimate considerably, but most underestimate slightly, most individual's may underreact to the threat of COVID-19 even though as a population their average estimate is accurate. More importantly, Laypeople aren't usually asked to predict the spread of the virus; what is more relevant is how these graphs affect their subjective perception of threat and need for response ${ }^{\mathrm{vi}}$. This is what we look at next: how does choice of scale change laypeople's overall subjective judgments of growth and threat, and resulting need for action in response to Coronavirus?

\section{S2: Beliefs and attitudes}

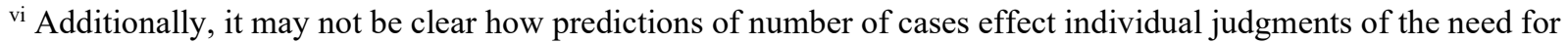
action. For example, individuals may be more concerned with the total number of cases they believe there will be in the near future, or they may be more concerned with the increases in the growth rate of cases. This is one of the reasons we choose to measure participant judgments of growth, threat, and resulting need for action and policy response directly.
} 
LOGARITHMIC GRAPHS DISTORT JUDGMENT

In Study 2 we test how the two presentations of disease data affect beliefs about the disease's

growth and threat, and whether this translates into attitudes towards individual and government response.

Participants. 891 MTurk workers $\left(\mathrm{M}_{\mathrm{age}}=37.9\right.$, 48\% female $)$ completed the study ${ }^{\mathrm{vii}}$.

Procedure. Subjects were presented with disease data for four different countries in succession in either log or linear formats and asked questions about each. Finally, because it is possible that individuals are more accurate when the data is embedded in a larger context ${ }^{14}$, we also varied whether participants saw only data of the case prevalence of the target country, or saw this data on the same graph as data for 10 additional countries.

Results. Judgments of growth, danger, appropriate policy response, and required individual effort to combat Coronavirus were all lower when logarithmic scales were used, regardless of the number of countries presented (figure 4). Alarmingly, relative to the linear condition, participants who saw logarithmic graphs indicated that they would wear masks less often and be less committed to social distancing. Presenting additional countries for context reduced perception of severity, likely due to these countries' high case counts, however it did not moderate the difference in judgments between logarithmic and linear scales. Summaries alongside Studies 1B and 3 are presented in figure 5.

\footnotetext{
${ }^{\text {vii }}$ A total of 921 individuals participants in this study with $4 \%$ failing the attention check. Per our preregistration we removed these individuals for analysis, but results remain the same if they are included.
} 
LOGARITHMIC GRAPHS DISTORT JUDGMENT

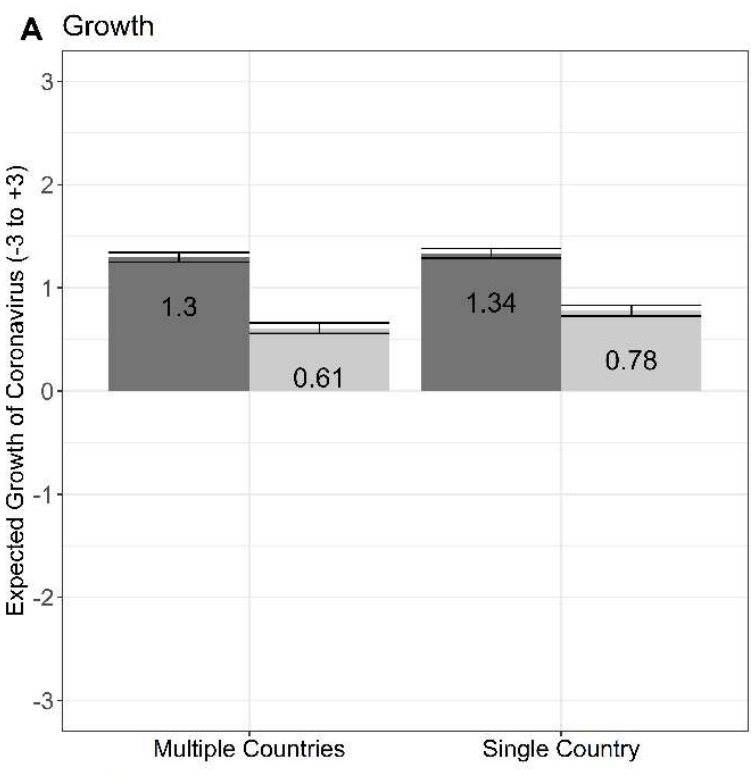

B Danger
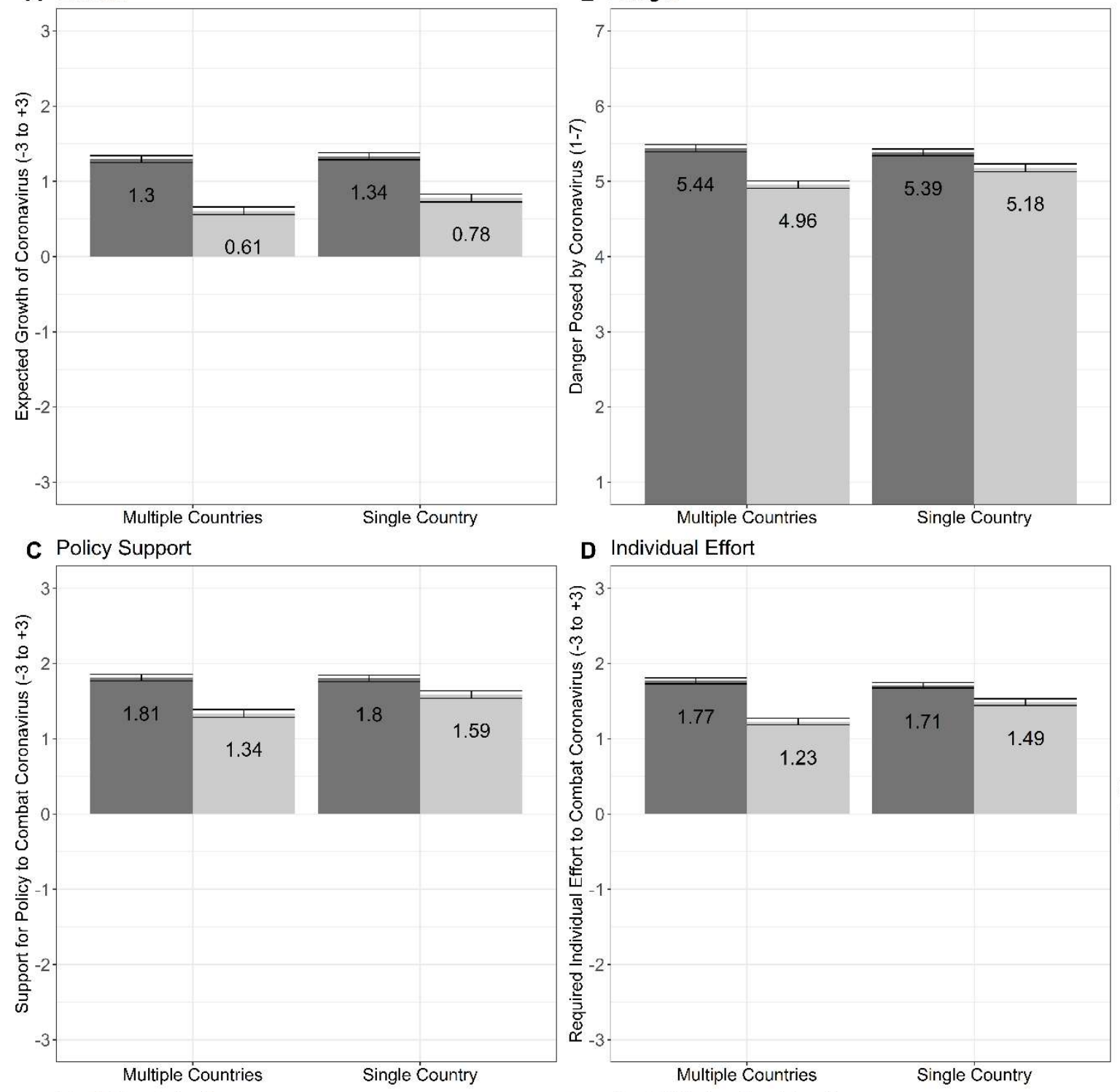

D Individual Effort
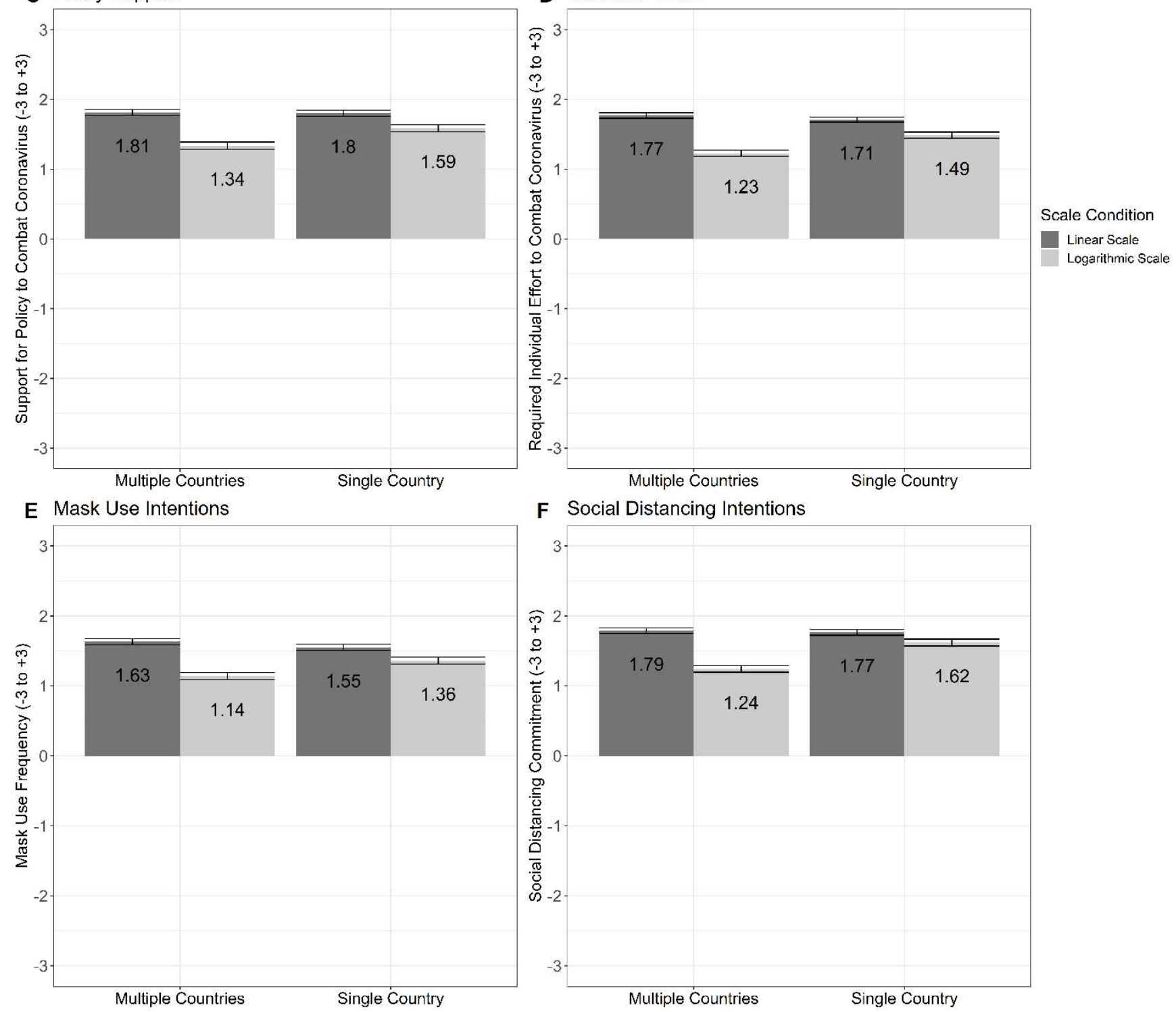

F Social Distancing Intentions

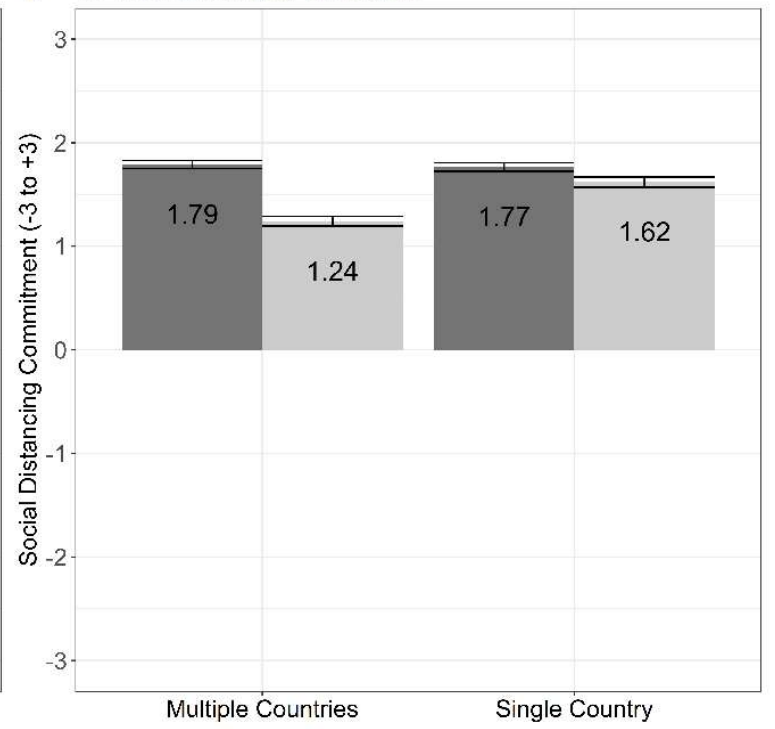


LOGARITHMIC GRAPHS DISTORT JUDGMENT

Figure 3. Judgments of growth (A), danger (B), need for policy response by governments $(C)$, and required individual effort (D), as well as behavioral intentions to use masks (E) and socially distance (D) by each condition. Figure $3 A$ shows mean responses to the question "Based on this graph, how do you expect the growth rate of cases, i.e. the number of new cases per day, to change in [COUNTRY]?", with responses anchored on the left at "(-3) Significantly fewer new cases/day” and on the right with "(3) Significantly more new cases/day". Figure 3B: "Based on this graph, how dangerous do you believe Coronavirus is to [COUNTRY] and its citizens?", from "(1) Not at all dangerous", and on the right with “(7) Extremely dangerous”. Figure 3C: "Based on this graph, how much do you agree with the policy: '[COUNTRY] should ban public gatherings, close non-essential businesses, and ask all citizens to stay at home unless they are going to work or carrying out necessary errands. "“, from "(-3) Disagree strongly” to "(3) Agree strongly". Figure 3D: "Think of all the efforts the people in [COUNTRY] may be doing to try to stop the disease, such as social distancing, wearing face masks, and avoiding non-essential travel. Based on this graph, how do you think people in [COUNTRY] should change the amount of effort they put into these actions?", from "(-3) Significantly decrease effort” to "(3) Significantly increase effort”.

Figure 3E: US-only question, "Based solely on this graph, do you see yourself wearing a mask more or less often than you do now, from "(-3) Significantly less often” to "(3) Significantly more often. Figure 3F: US-only question, "Based solely on this graph, do you see yourself being significantly more or less careful about social distancing relative to now", with scale anchors identical to Figure $3 E$.

\section{S3: Debiasing}

In recent weeks, outlets began educating their audience on how to correctly interpret logarithmic graphs. In this study we evaluate if this education makes interpretations of growth and threat of the virus from logarithmic graphs more in line with those from linear graphs. 
LOGARITHMIC GRAPHS DISTORT JUDGMENT

Participants. $739\left(\mathrm{M}_{\mathrm{age}}=40.3\right.$ years, 50.2\% female) MTurk workers completed the study ${ }^{\mathrm{viii}}$.

Procedure. Study 3 had a 2 (logarithmic vs. linear axis) by 2 (debiasing intervention vs. control) between-subjects design. The debiasing intervention was an approximately two-minute-long clip from a video made by Vox ${ }^{\mathrm{ix}}$ explaining how to correctly interpret COVID-19 graphs.

While informing participants about the pitfalls of logarithmic graphs reduced the difference in perceptions of growth and danger, perceptions of growth and danger were still significantly lower. Education helped decrease the effects of logarithmic scales on judgment, but did not eliminate $\mathrm{it}^{\mathrm{x}}$. Summaries alongside Studies $1 \mathrm{~B}$ and 2 are presented in figure 5.
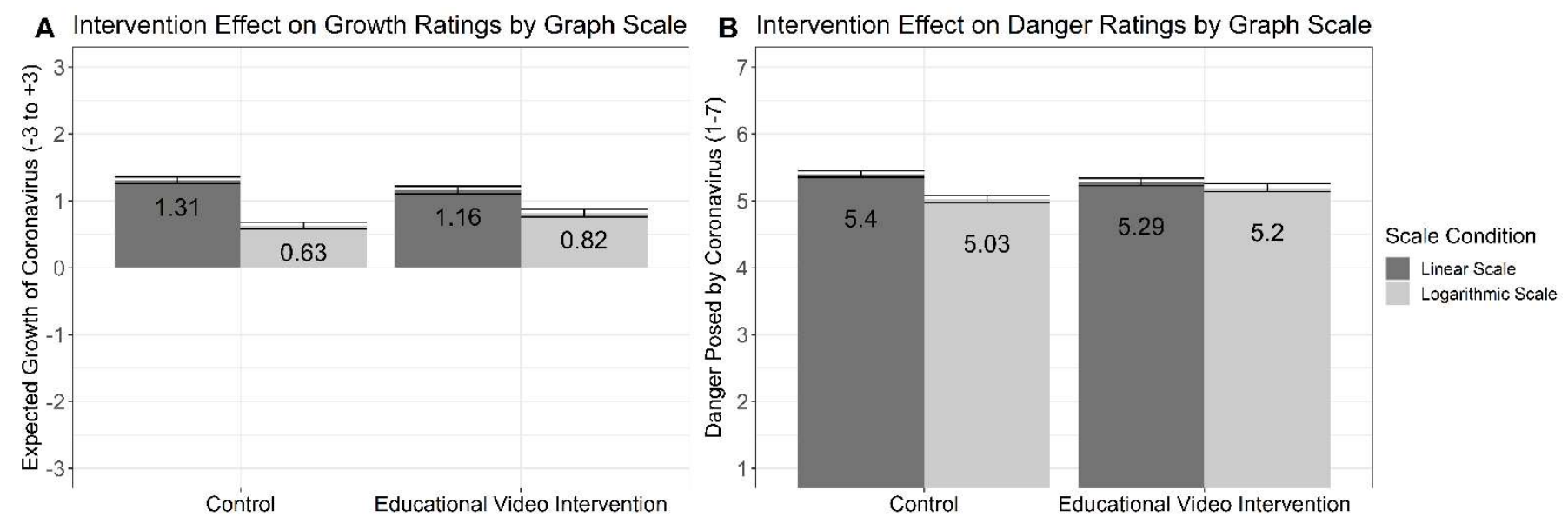

Figure 4. Growth (A, left) and Danger (B, right) ratings for both the educational intervention and control conditions by axis scale condition. Figure $4 A$ and $4 B$ show mean responses to questions identical to those asked in Figure $3 A$ and $3 B$ respectively.

\section{General Discussion}

\footnotetext{
viii 910 workers completed the study, and 739 (82\%) remained after exclusions. All results remain significant without exclusions

${ }^{\text {ix }}$ https://www.youtube.com/watch?v=O-3Mlj3MQ_Q

${ }^{x}$ The fact that when individuals better understand logarithmic graphs their estimates of threat and growth become closer to their estimates when they see linear graphs implies that these higher judgments of threat may better represent the positions individuals would endorse with fuller information they can understand more clearly.
} 
LOGARITHMIC GRAPHS DISTORT JUDGMENT

In these studies, we found consistent evidence that the public formed less accurate impressions of the current COVID-19 situation when data was presented using a logarithmic scale as compared to a linear scale. Logarithmic scales also led participants to believe the pandemic was less severe and less dangerous, as summarized in figure 5. Individuals presented with logarithmic graphs were less supportive of government policies aimed at reducing the growth of the virus, and less likely to take individual actions such as mask-wearing and social distancing.
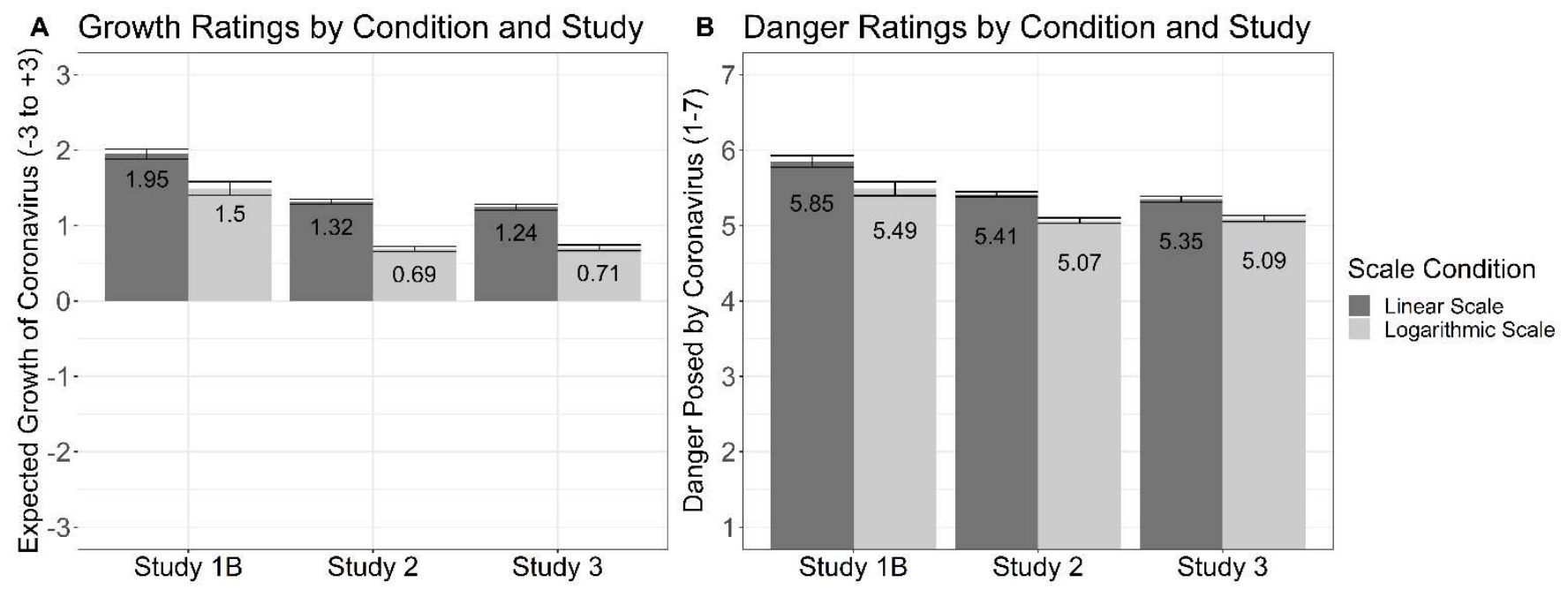

Figure 5. Averages of participants ratings of disease growth and danger by condition (linear vs.

logarithmic axis) for Studies $1 B$, 2, and 3. Figure 5A and 5B show responses to questions identical to questions asked in figures $3 A$ and $3 B$ respectively.

We also tested common features of situations and individuals which are typically believed to reduce these kinds of biases. We found that providing additional data on other countries did not reduce the differences between the two types of graphs, and that educating participants reduced, but did not eliminate these differences. We did not establish that increased inaccuracy in predictions of cases due to viewing graphs with logarithmic axes directly lead to any behavior change - these two effects appear to possibly be independent, but future work should examine this in more detail. 
LOGARITHMIC GRAPHS DISTORT JUDGMENT

One feature we did not extensively test, is whether the actual growth of the outbreak could influence the difference in perception between the two types of graphs. To illustrate, figure 6A shows the first 20 days of the outbreak in logarithmic and linear format, while figure $6 \mathrm{~B}$ also includes the next 30 days. As can be seen, the two graphs look more similar in figure 6A, when growth-rates are still climbing, than in figure $6 \mathrm{~B}$, when they have stabilized or decreased. To test whether the actual pattern of the outbreak could moderate the effects documented in this manuscript, we ran a simple conceptual replication of Study 2, only this time choosing countries whose growth fit one of the two patterns in figure 6 (see Appendix Study 1). We found that the effects of logarithmic axes existed regardless of the slope of the actual growth.
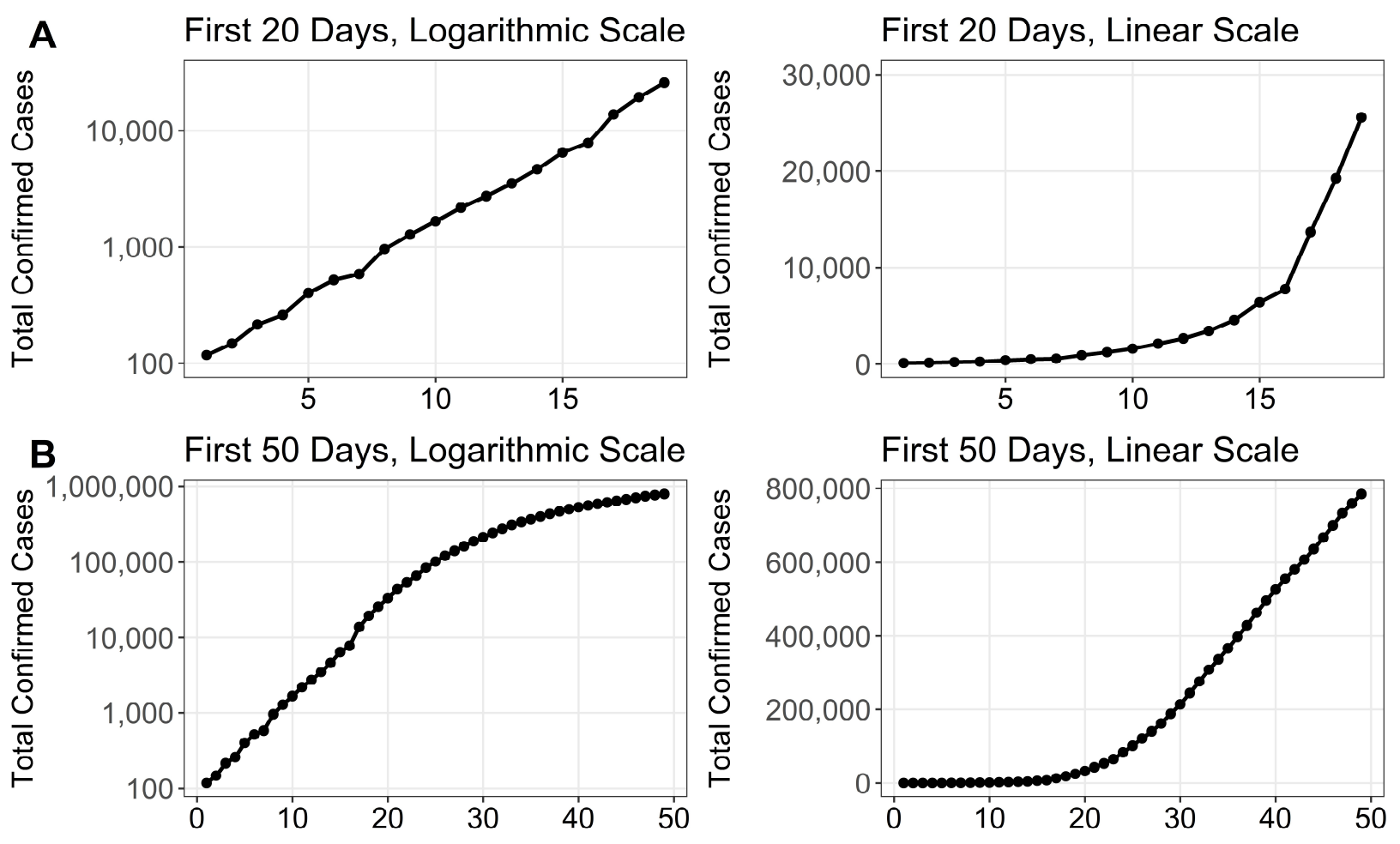

Figure 6. Total confirmed cases of Coronavirus in the United States by the number of days since 100 confirmed cases were reached, plotted over the first 20 days (A, top) and first 50 days (B, bottom). 


\section{LOGARITHMIC GRAPHS DISTORT JUDGMENT}

In two of our studies, we also tested for several individual differences that could influence the degree to which individuals may struggle with logarithmic graphs. Surprisingly, we found in both studies that higher objective numeracy does not result in a reduction of the difference between logarithmic and linear scales, but that this difference is sometimes higher for more numerate individuals. More information on all individual difference measures can be found in the appendix.

The skeptical reader may argue that we may have stacked the deck against logarithmic scales. Afterall, the predominant benefits of using a logarithmic scale focus on growth-rate and growthcomparison, while we examined impressions of risk and danger. We would argue that comparing growthrates between countries may be important for epidemiologists and politicians who are in a position to implement and evaluate high-level policies, but for a regular audience it is more important that they accurately estimate risk and respond accordingly. That said, to take this argument seriously, we ran a study in which we expected logarithmic graphs may be superior. However, the claimed advantages of logarithmic graphs did not appear; we found that, for a lay audience, logarithmic graphs did not actually improve accuracy of comparative growth judgments (see Appendix Study 2).

\section{Recommendations for journalists and scientists}

These findings make it clear that journalists' and scientists' choices to use either logarithmic or linear axis graphs matter, and can influence public response to COVID-19. Overall, these studies indicate that when people are presented with graphs using logarithmic, instead of linear, axes they make less accurate predictions of future growth, view COVID-19 as less of a threat, and accordingly are less supportive of governmental and individual action against COVID-19. Education can reduce these effects, but not eliminate them. Logarithmic graphs still have a significant place in presenting scientific data. However, based on this research it is recommended to use them only when they are truly appropriate, to spend significant time explaining how to read the presented graph, and to supplement data presented in logarithmic graphs with linear graphs as well. 
LOGARITHMIC GRAPHS DISTORT JUDGMENT

\section{References}

1. Chapman, G. B., \& Coups, E. J. (2006). Emotions and preventive health behavior: Worry, regret, and influenza vaccination. Health Psychology, 25(1), 82-90.

https://doi.org/10.1037/0278-6133.25.1.82

2. Yan, Wudan. (2020, April 14). Coronavirus Tests Science's Need for Speed Limits. The New York Times, retrieved from https://www.nytimes.com/2020/04/14/science/coronavirusdisinformation.html

3. Lipkus, I. M., Samsa, G., \& Rimer, B. K. (2001). General Performance on a Numeracy Scale among Highly Educated Samples. Medical Decision Making, 21(1), 37-44. https://doi.org/10.1177/0272989x0102100105

4. Wagenaar, W. A., \& Sagaria, S. D. (1975). Misperception of exponential growth. Perception \& Psychophysics, 18(6), 416-422. https://doi.org/10.3758/bf03204114

5. Wagenaar, W. A., \& Timmers, H. (1979). The pond-and-duckweed problem; Three experiments on the misperception of exponential growth. Acta Psychologica, 43(3), 239-251. https://doi.org/10.1016/0001-6918(79)90028-3

6. Schwartz, L. M. (1997). The Role of Numeracy in Understanding the Benefit of Screening Mammography. Annals of Internal Medicine, 127(11), 966. https://doi.org/10.7326/0003-4819127-11-199712010-00003

7. Zikmund-Fisher, B. J., Smith, D. M., Ubel, P. A., \& Fagerlin, A. (2007). Validation of the Subjective Numeracy Scale: Effects of Low Numeracy on Comprehension of Risk 
LOGARITHMIC GRAPHS DISTORT JUDGMENT

Communications and Utility Elicitations. Medical Decision Making, 27(5), 663-671.

https://doi.org/10.1177/0272989x07303824

8. Peters, E., Hibbard, J., Slovic, P., \& Dieckmann, N. (2007). Numeracy Skill And The

Communication, Comprehension, And Use Of Risk-Benefit Information. Health Affairs, 26(3),

741-748. https://doi.org/10.1377/hlthaff.26.3.741

9. Frederick, S. (2005). Cognitive Reflection and Decision Making. Journal of Economic

Perspectives, 19(4), 25-42. https://doi.org/10.1257/089533005775196732

10. Zacks, J., \& Tversky, B. (1999). Bars and lines: A study of graphic communication. Memory \& Cognition, 27(6), 1073-1079. https://doi.org/10.3758/bf03201236

11. Beattie, V., \& Jones, M. J. (2002). The Impact of Graph Slope on Rate of Change Judgments in Corporate Reports. Abacus, 38(2), 177-199. https://doi.org/10.1111/1467-6281.00104

12. Cleveland, W. S., \& McGill, R. (1987). Graphical Perception: The Visual Decoding of Quantitative Information on Graphical Displays of Data. Journal of the Royal Statistical Society. Series A (General), 150(3), 192. https://doi.org/10.2307/2981473

13. Fischer, H., Schütte, S., Depoux, A., Amelung, D., \& Sauerborn, R. (2018). How Well Do COP22 Attendees Understand Graphs on Climate Change Health Impacts from the Fifth IPCC Assessment Report? International Journal of Environmental Research and Public Health, 15(5), 875. https://doi.org/10.3390/ijerph15050875

14. Wagner, S. H., \& Goffin, R. D. (1997). Differences in Accuracy of Absolute and Comparative Performance Appraisal Methods. Organizational Behavior and Human Decision Processes, 70(2), 95-103. https://doi.org/10.1006/obhd.1997.2698 